\title{
NANO SELF-ASSEMBLED ION-SENSITIVE FIELD-EFFECT TRANSISTORS FOR ACETYLCHOLINE BIOSENSING
}

\author{
Yi Liu, Arthur G. Erdman, and Tianhong Cui" \\ Department of Mechanical Engineering, University of Minnesota \\ Minneapolis, MN 55455, USA \\ Tel: 612-626-1636 Fax: 612-625-6069 E-mail: tcui@me.umn.edu
}

\begin{abstract}
In this paper, the fabrication and characterization of acetylcholine biosensors based on nano self-assembled ion-sensitive field-effect transistors (ISFETs) are demonstrated. The fabrication is implemented with a very low-cost layer-by-layer nano self-assembly technique. A self-assembled polyaniline thin film works as the semiconducting channel material, while a $\mathrm{SiO}_{2}$ nanoparticle thin film serves as the gate dielectric material. A typical polyaniline ISFET operates at a low-voltage range and has a mobility of $1.49 \mathrm{~cm}^{2} / \mathrm{Vs}$. Acetylcholine in a concentration as low as $1 \mu \mathrm{M}$ could be detected with this sensor. The results presented herein suggest a route to inexpensive ion-sensitive field-effect transistors for biosensing applications.
\end{abstract}

\section{INTRODUCTION}

Acetylcholine (Ach) is a chemical transmitter in both the peripheral nervous system and central nervous system of many organisms including humans. Acetylcholine neurotransmission is considered to play a critical role in human during the processes such as behavioral activity, arousal, attention, learning, memory, etc. An abnormally short supply of acetylcholine is associated with Alzheimer's disease, which ranks the fourth in the causes of death among adults. Therefore, the sensing of acetylcholine concentration is important and of great interest. Some methods can be used to detect the concentration of acetylcholine, for example, electrospray ionization mass spectrometry (EIMS) [1] and biosensors [2]. However, both the EIMS and the silicon-based biosensors are relatively expensive because the EIMS is a bulky instrument and the fabrication of silicon-based biosensors is complicated and costly. Polymers and nanoparticles are alternatives to silicon for making low-cost electronic devices and systems. In this report, polymer- and nanoparticle-based field-effect transistors are fabricated and characterized for acetylcholine biosensing.

Biosensors are analytical devices incorporating a biological material with a physical transducer or transducing microsystem. The transduction mechanism may be optical, electrical, thermal, piezoelectric, magnetic, etc. One of the most popular biosensors is based on the ion-sensitive field-effect transistor (ISFET), which was first introduced by Bergveld in 1972 [3]. The advantages of ISFET based biosensors include high sensitivity, real-time, and label-free detection of a wide range of chemical and biological species. Since ISFET biosensors can be produced by the integrated circuit production method, they could be reduced in size and mass-produced. They are not only known as one of the most important miniaturized biosensors, but also as devices to bridge the gap between microelectronics and biotechnology.

Recently, layer-by-layer (LBL) nano self-assembly technique has attracted much attention since the introduction by G. Decher et al. [4] The LBL process involves alternating immersion of a substrate into aqueous solutions of polycations and polyanions. With each immersion, a polyion layer is deposited and the surface ionization of the substrate is reversed, allowing a subsequent layer with opposite charge to be deposited. Multilayer thin films made of interesting charged materials such as nanoparticles, conjugated polymers, DNA, and proteins, etc. can be formed with controlled thickness in nanometer scale. Using LBL self-assembly, it is very simple and inexpensive to produce organized films similar to the ones obtained with the sophisticated and expensive molecular beam epitaxy technology. The versatility, relative ease of preparation, ultra low cost, and potential for scale-up have made LbL self-assembly a viable chemical approach for the fabrication of nanostructure devices. There are several self-assembly techniques, such as langmuir-blodgett (LB) films, self-assembly of monolayers (SAM), and LBL self-assembly. Compared to LB technique and SAM technique, the LBL films exhibit a much larger thermal and mechanical stability, and can be prepared up to hundreds of layers. LBL self-assembly is proving to be a useful and versatile technique for the formation of multilayered thin films with a wide range of electrical, optical, and biological properties. It can find applications including optical diode [5], biosensor [6], etc.

Previously, we have reported the fabrication of LBL self-assembled nanoparticle thin films as building blocks for a field-effect transistor [7]. In this paper, the LBL self-assembly technique is extended to form thin films of polymer, nanoparticle and enzyme for the fabrication of ISFET based acetylcholine-sensitive biosensor. Conjugated polymer, polyaniline (PANI), has been self-assembled as the semiconductor channel material. Figure 1 shows the schematic diagram of the acetylcholine-sensitive ISFET. The sensing principle of the acetylcholine ISFET biosensor is based on the Hilton Head Island, South Carolina, June 4-8, 2006 
biocatalyzed hydrolysis of acetylcholine in the presence of acetylcholine esterase (AchE), as shown in Eq. (1). The hydrolysis results in acetic acid that changes the $\mathrm{PH}$ of the solution, and can be detected by the ISFET.

$$
\begin{aligned}
& \left(\mathrm{CH}_{3}\right)_{3} \mathrm{~N}^{+} \mathrm{CH}_{2} \mathrm{CH}_{2} \mathrm{OCOCH}_{3}+\mathrm{H}_{2} \mathrm{O} \stackrel{\text { AchE }}{\longrightarrow} \\
& \left(\mathrm{CH}_{3}\right)_{3} \mathrm{~N}^{+} \mathrm{CH}_{2} \mathrm{CH}_{2} \mathrm{OH}+\mathrm{HOCOCH}_{3}
\end{aligned}
$$

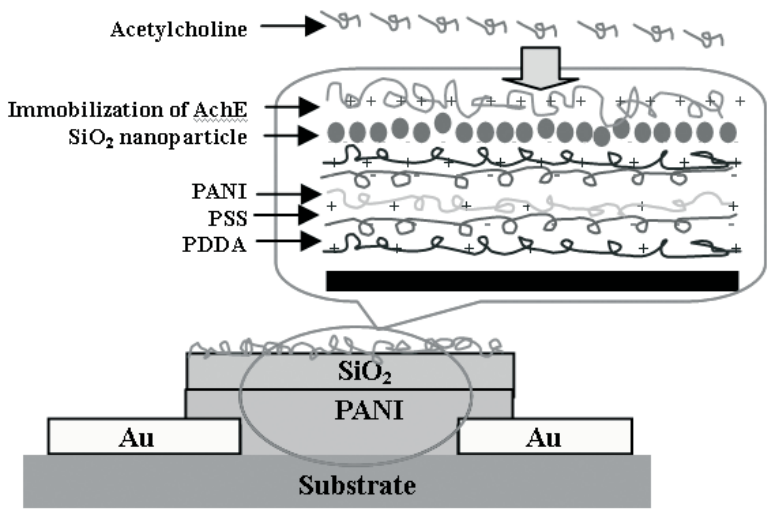

Figure 1. The schematic diagram of the Ach-sensitive ISFET

\section{EXPERIMENT}

The polyions involved in the fabrication process were positively charged poly(dimethyldiallyl-ammonium chloride) (PDDA) and negatively charged poly(styrenesulfonate) (PSS). Polyaniline doped with sulfuric acid was used as the channel material. $\mathrm{SiO}_{2}$ nanoparticles $40 \sim 60 \mathrm{~nm}$ in diameter were used as the gate dielectric material. The acetylcholine esterase from electric eel and acetylcholine chloride were prepared from de-ionized (DI) water. All the above materials were purchased from Sigma-Aldrich Corporation, except that the $\mathrm{SiO}_{2}$ nanoparticle dispersion was from Nissan Chemical Corporation.

The nano self-assembled ISFETs were built on a standard 4-inch silicon wafer with a layer of $\mathrm{SiO}_{2} 300 \mathrm{~nm}$ thick. First, two layers of chrome $(100 \mathrm{~nm})$ and gold $(200 \mathrm{~nm})$ were deposited on the wafer with e-beam evaporation, and then patterned by optical lithography to form the source/drain electrodes. Next, photoresist was spin-coated on the wafers and patterned to open windows directly above the channel region. Prior to the layer-by-layer self-assembly, the wafer were put into the $\mathrm{O}_{2}$ plasma for 30 seconds to clean the photoresist residue inside the opening window. The wafer were then alternately immersed in aqueous PDDA and PSS solutions, in a sequence of [PDDA (10 $\mathrm{min})+$ PSS $(10 \mathrm{~min})]_{3}$. These three bi-layers of PDDA/PSS served as the precursor layers that helped to enhance the subsequent adsorption of conjugated polymers and nanoparticles. PDDA and PSS also worked as the sandwich layers between two neighboring nanoparticle, polymer, or AchE layers, depending on the charge polarization of the solution. Between two immersions of the wafer into solutions, there was intermediate rinsing using DI water for 1 minute to remove the residue on the previous layer from the surface. Following the precursor layers, five layers of PANI polymer thin films were coated on the entire wafer surface in the sequence of [PANI (10 min $)+$ PSS $(10 \mathrm{~min})]_{5}$. Next, the $\mathrm{SiO}_{2}$ nanoparticle dispersion were coated in the sequence of $\left[\mathrm{PDDA}(10 \mathrm{~min})+\mathrm{SiO}_{2}(4\right.$ $\mathrm{min})]_{6}$ to produce a gate dielectric thin film. Before immobilizing AchE on the top surface of $\mathrm{SiO}_{2}$ nanoparticle thin film, the wafer was soaked in an acetone solution with ultrasonic agitation to lift off the self-assembled materials not in the target window. Finally, AchE was immobilized on the top of the $\mathrm{SiO}_{2}$ film in the sequence of PDDA (10 min) + [PSS (10 $\left.\mathrm{min})+\operatorname{AchE}(10 \mathrm{~min})]_{3}\right\}$. Scanning electron microscopy (SEM) was used to investigate the self-assembled nanoparticle and AchE thin films. Figures $2 a$ and $2 b$ show images of self-assembled $\mathrm{SiO}_{2}$ nanoparticle thin films and AchE enzyme on the top of channel region.

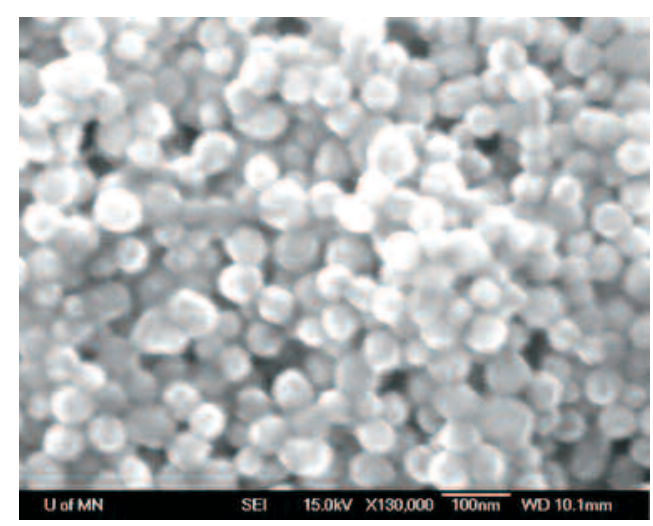

(2a)

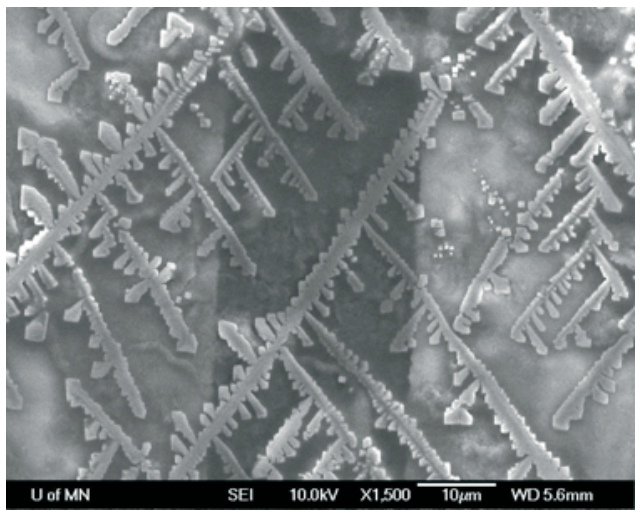

(2b)

Figure 2. The SEM images of (2a) $\mathrm{SiO}_{2}$ nanoparticle thin film, and (2b) AchE enzyme on the top of channel region.

\section{RESULTS AND DISCUSSIONS}

A HP 4156B semiconductor parameter analyzer is used to characterize the electrical behaviors of the fabricated ISFETs. $\mathrm{Ag} / \mathrm{AgCl}$ is used as the reference gate electrode. Figure 3a shows the output characteristic of a typical PANI ISFET at an acetylcholine solution with a concentration of $10 \mathrm{mM}$. This ISFET behaves like a traditional metal oxide semiconductor field-effect transistor (MOSFET). At higher positive gate 
voltage, the drain current is higher, which indicates that the LBL self-assembled PANI ISFET is functional as an n-type transistor. It is at the "normal-on" state at zero gate voltage, and then works at depletion mode with a negative bias. Electron polarons and bipolarons are the main charge carriers in the n-type PANI semiconductor thin films. This is different from the spin-coated PANI transistor that work as a p-type transistor [8][9]. These two different charge carrier mechanisms are due to the difference between the layer-by-layer self-assembled PANI thin film and the spin-coated PANI thin film. In the spin-coated PANI thin film, only the PANI solution was deposited on the channel region between the source and the drain electrodes. However, in the layer-by-layer self-assembled PANI, the PANI was sandwiched into the PSS polyelectrolyte by electrostatic force. The network of mixed PANI and PSS results in strong electron-hole asymmetry in PANI thin film. This n-type asymmetry with respect to polaronic effects, which has been predicted by J. Libert et al [10], can contribute to the n-type field-effect characteristics. The n-type field-effect in LBL self-assembled PANI thin films has also been observed by J. Paloheimo et al. [11]

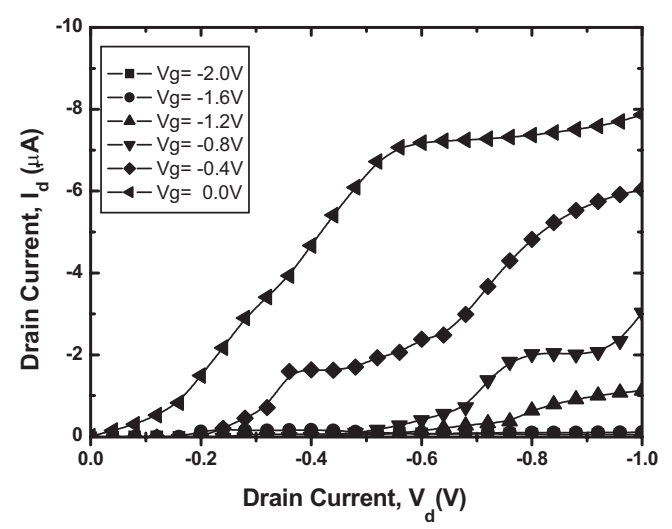

(a)

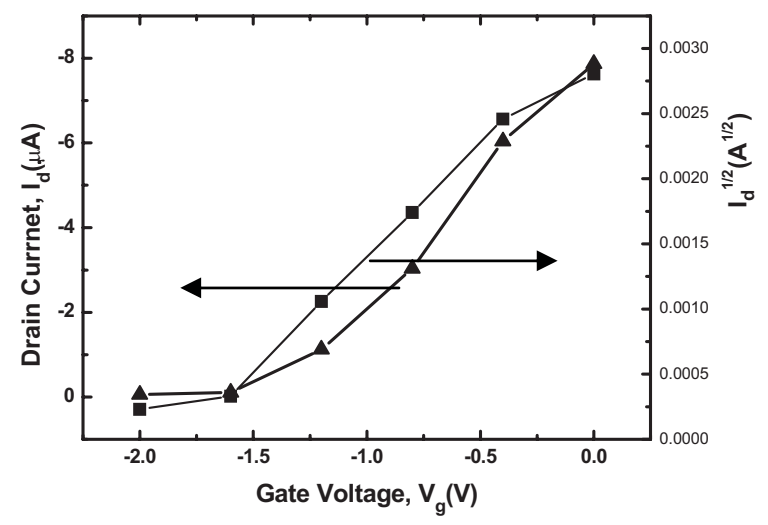

(b)

Figure 3. (a) The output characteristic and (b) transfer characteristic of the PANI ISFET in an acetylcholine solution with a concentration of $10 \mathrm{mM}$.

Figure $3 \mathrm{~b}$ shows the transfer characteristic of the same PANI ISFET in the acetylcholine solution $(10 \mathrm{mM})$. The extracted threshold voltage is $-1.8 \mathrm{~V}$. The calculated mobility based on the traditional MOSFET theory is $1.49 \mathrm{~cm}^{2} / \mathrm{Vs}$. There are 40 ISFETs fabricated on one wafer. The characterized mobility of these devices is within the range from 1 to $5 \mathrm{~cm}^{2} / \mathrm{Vs}$. It is noticed that the general polyaniline mobility is within the range from $10^{-5}$ to $10^{-4} \mathrm{~cm}^{2} / \mathrm{Vs}$ [11]. Our PANI based ISFET has a higher mobility, and were investigated carefully.

It is observed that the gate current is higher when the gate voltage is zero, but reduces rapidly when the gate voltage negatively increases. This implies that there are hydrogen ions passing through the gate dielectric layer and participating in the charge transport at zero or low negative gate bias. Since the diameter of the hydrogen ion is less than $0.3 \mathrm{~nm}$, it can easily penetrate the small spaces among the $\mathrm{SiO}_{2}$ nanoparticles. Since polyions (PDDA and PSS) within the multilayer films are also conductive in solution, the total charge carriers in the channel should include hydrogen ions, electron polarons from the gate-affected PANI semiconductor thin films and the polyions between the PANI thin films. These carriers greatly increase the channel's conductivity as well as the charge transport ability. Therefore, the calculated mobility should be an "equivalent mobility" that takes into account all the charge carriers. The equivalent mobility could be much higher than a similar transistor in atmosphere ambient. Similar behavior is also observed by Sandberg, et al. in an all-polymer field-effect transistor, which has a high mobility $\left(>100 \mathrm{~cm}^{2} / \mathrm{Vs}\right)$ in a humid environment, about $4 \sim 5$ orders higher than a typical polymer transistor using poly(3-hexylthiophene) as channel material [12].

Figure 4 shows the sensitivity of a typical PANI ISFET for different concentrations of acetylcholine when the gate voltage is $0 \mathrm{~V}$ and the drain voltage is $-1 \mathrm{~V}$. At lower concentration of acetylcholine, the drain current is also smaller. This is because lower concentration of hydrogen ions can be generated during the reaction between acetylcholine and acetylcholine esterase when the concentration of acetylcholine is lower. This has also demonstrated how the device can be effectively used as a biosensor. The ISFET could detect a concentration change of acetylcholine down to $1 \mu \mathrm{M}$.

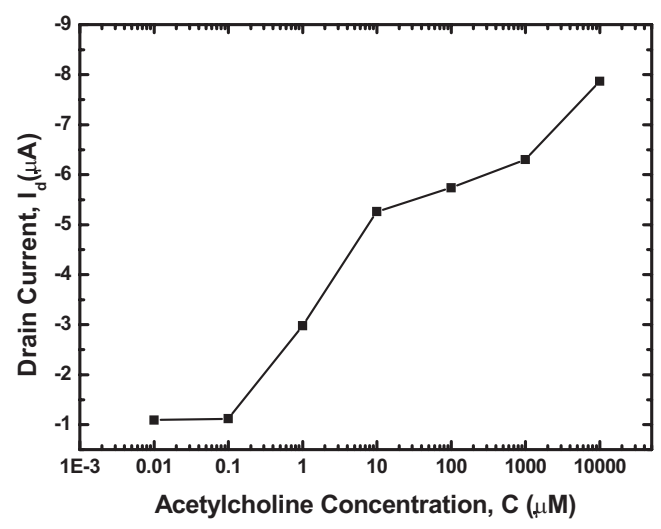

Figure 4. The sensitivity of the PANI ISFET for sensing acetylcholine concentration. 
Figure 5 shows the response time of the PANI ISFET with respect to the open circuit potential change for differrent concentrations of acetylcholine. The voltage between drain electrode and the $\mathrm{Ag} / \mathrm{AgCl}$ reference electrode escalated with an increased acetylcholine concentration. When the concentration of acetylcholine was changed from $10 \mathrm{nM}$ to $100 \mathrm{nM}$, the change of gate potential was negligible. However, when the concentration of acetylcholine was $1 \mu \mathrm{M}$, the increase of the gate potential was obvious. Further increases in the concentration of acetylcholine also greatly increased the gate potential. Since a change of the gate voltage will affect the channel conductivity, it is obvious that the ISFET could detect variations of acetylcholine concentration larger than $1 \mu \mathrm{M}$.

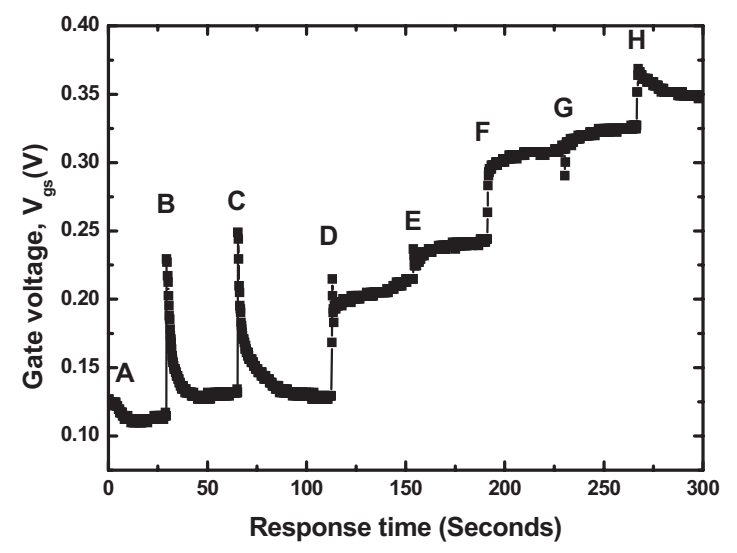

Figure 5. The response time of the PANI ISFET at different concentration of acetylcholine (A: DI water, B: $10 \mathrm{nM}, \mathrm{C}: 100$ $n M, D: 1 \mu M, E: 10 \mu M, F: 100 \mu M, G: 1 \mathrm{mM}, H: 10 \mathrm{mM})$.

\section{CONCLUSIONS}

In this paper, polyaniline ISFET based on the low-cost layer-by-layer nano self-assembly was successfully fabricated and used for the acetylcholine biosensing. Acetylcholine concentration down to $1 \mu \mathrm{M}$ could be detected use the self-assembled biosensors. The ISFET exhibited high performance, operating at very low voltages with a high mobility. The results show promise for a large scale logic circuit integration of biosensors and biomedical systems. Further work is ongoing to improve the sensitivity of the ISFET for acetylcholine sensing such that it could detect acetylcholine down to $10 \mathrm{nM}$.

\section{ACKNOWLEDGEMENT}

The authors would like to thank Prof. David Redish from Department of Neuroscience at the University of Minnesota for his helpful discussion.

\section{REFERENCES}

[1]. R. Dunphy and D.J.Burinsky, "Detection of choline and acetylcholine in a pharmaceutical preparation using high-performance liquid chromatography/electrospray ionization mass spectrometry", Journal of Pharmaceutical and Biomedical Analysis, 31, 905 (2003).

[2]. A.B. Kharitonov, M. Zayats, A. Lichtenstein, E. Katz, and I. Willner, "Enzyme monolayer-funcitionalized field-effect transistors for biosensor applications", Sensors and Actuators B, 70, 222, (2000).

[3]. P. Bergveld, "Development, operation and application of the ion sensitive field-effect transistor as a tool for electrophysiology", IEEE Trans. Biomed. Eng. BME-19, 342, (1972).

[4]. G. Decher, "Fuzzy Nanoassemblies: Toward layered polymeric multicomposites", Science, 277, 1232 (1997).

[5]. A. A. Mamedov, A. Belov, M. Giersig, N. N. Mamedova, and N. A. Kotov, "Nanorainbows: Graded Semiconductor Films from Quantum Dots", J. Am. Chem. Soc., 123, 7738, (2001).

[6]. Q. Chen, J. Han, H. Shi, B. Wu, X. Xu, and T. Osa, "Use of Chitosan for developing layer-by-layer multilayer thin films containing glucose oxidase for biosensor applications", Sensor Letters, 1, 102, (2004).

[7]. T. Cui, Y. Liu, and M. Zhu, "Field-Effect Transistors with Layer-by-Layer Self-Assembled Nanoparticle Thin Films as Channel and Gate Dielectric", Applied Physics Letters, 87, 183105, (2005).

[8]. C. Kuo, S. Chen, G. Hwang, H. Kuo, "Field-effect transistor with the water-soluble self-acid-doped polyaniline thin films as semiconductor," Synthetic Metals, 93, 155, (1998).

[9]. C. Kuo, S. Weng, R. Huang, "Field-effect transistor with polyaniline and poly(2-alkylaniline) thin film as semiconductor," Synthetic metals, 88, 101, (1997).

[10]. J. Libert, J. L. Bredas, and A.J. Epstein, "Theoretical study of $\mathrm{p}$ - and n-type doping of the leucoemeraldine base form of polyaniline: Evolution of the geometric and electronic structure", Phys. Rev. B. 51, 5711, (1995).

[11]. J. Paloheimo, K. Laakso, H.Isotalo, H. Stubb, "Conductivity, thermoelectric power and field-effect mobility in self-assembled films of polyaniline and oligoanilines", Synthetic metals, 68, 249, (1995).

[12]. H. Sandberg, T.G. Backlund, R.Osterbacka, and H. Stubb, "High-performance all-polymer transistor utilizing a hygroscopic insulator", Advanced Materials, 16, 1112, (2004). 